\title{
LA MOVILIZACIÓN DE CONOCIMIENTOS EN LA SOCIEDAD: UN RETO PARA LOS PROGRAMAS DOCTORALES EN LA UNIVERSIDAD PÚBLICA LATINOAMERICANA
}

The mobilization of knowledge in society: A challenge for doctoral programs in the Latin American public University

\section{Luis A. Ordóñez Vela*}

Resumen: Los programas doctorales son particularmente adecuados para apoyar el proceso de cambio paradigmático de la organización universitaria e incrementar la eficiencia requerida para el procesamiento del conocimiento disponible, y el nuevo a ser creado, en función de proveer de evidencias a los tomadores de decisiones en la sociedad, al convertir la movilización de conocimientos desde la universidad en un problema de la organización universitaria, que no de los profesores o del personal administrativo. En el presente trabajo revisamos las disonancias cognoscitivas que detectamos en nuestra normativa general para estudios de postgrado y cómo la estrategia pedagógica de los Cursos Abiertos Masivos En Línea (CAMEL) puede ser aprovechada para profundizar el nuevo paradigma. En el proceso, revisamos las dificultades más frecuentemente encontradas en la implementación de los CAMEL en tres ámbitos diferentes, los programas doctorales en Ciencias Sociales, Educación y Administración y Gerencia de dos universidades venezolanas. Encontramos que la mayor dificultad se presenta en el terreno de las culturas colaborativas requeridas para obtener la generación de equipos eficientes en nuestro medio,

Presidente de la Fundación Interconectados y docente universitario. 
unido a la necesidad de aplicar una adecuada re-ingeniería que permita el nuevo tipo de organización, donde el nuevo tipo de egresado al más alto nivel académico (los nuevos doctores) y los nuevos egresados universitarios en su rol de "colonizadores" de la sociedad, logren restablecer el vínculo perdido entre ambos mundos, la universidad y la sociedad. Encontramos que la mayor dificultad se presenta en el terreno de las culturas colaborativas requeridas para obtener la generación.

Palabras clave: Movilización, conocimiento, sociedad, programa doctoral, universidad pública, organización universitaria, postgrado, CAMEL.

Abstract: Doctoral programs are particularly suitable to support the paradigm shift required by the university organization in order to increase its efficiency for processing available and the "new to be created" knowledge, based on providing evidence to decision makers in society, by shifting the universities knowledge mobilization from a problem of teachers or staff to a problem of the University organization. In this paper we review the cognitive dissonance that we detect in our general rules for postgraduate studies and how the educational strategy of the Massive Open On-line Courses (MOOC's) can be used to support the new paradigm. In the process, we review the difficulties, most frequently encountered, in the implementation of MOOC's in three different fields, doctoral programs in social sciences, education and administration and management from two Venezuelan universities. We find that the main difficulty arises in the field of the collaborative culture required for the generation of efficient teams in our academic environment, coupled with the need for a proper re-engineering that will allow the new type of organization, where the new type of graduate at the highest academic level (the new doctors) and the new university graduates in their role of "settlers of society" will restore the link between both worlds, the University and society.

Keywords: Mobilization, knowledge society, doctoral program, public college, university organization, graduate, CAMEL. 
La movilización de conocimientos en la sociedad: Un reto para los programas doctorales en la universidad pública latinoamericana

\section{Introducción}

La forma como una sociedad moviliza información (conocimiento) para la toma de decisiones es cultural-dependiente. Los estudios desde la antropología, la psicología, la administración y la sociología parecieran sugerir que la gestión del conocimiento va a depender de la cultura de la sociedad o institución inmersa en esa sociedad. Así, la universidad como organización ${ }^{1}$ inmersa en una sociedad determinada no va a escapar a este problema.

Pierre Bourdieu habló de la existencia de espacios sociales diferenciados y estancos (Martínez García, 1998). La universidad pública venezolana, un caso particular de la universidad pública latinoamericana, pudiese haber generado su propio campo, con un babitus característico, que la diferencia y la hace entrar en procesos de violencia simbólica con otros campos de la sociedad, en particular con el campo político. Se genera así una situación en la que la intermediación de la información necesaria para la toma de decisiones, ya de por sí un proceso complejo, se dificulta mucho más, agravado por el hecho de que no cuenta nuestra universidad con estructuras que "colectivamente" detecten información, la interpreten y traduzcan adecuadamente a los decisores. En otras palabras, las estructuras resultantes del actual paradigma universitario que ve la universidad como generadora, transmisora y extensionista del conocimiento, no resultan suficientes para movilizar conocimiento eficientemente en nuestra sociedad (Ordóñez, 2015).

\footnotetext{
A todo lo largo de este ensayo entenderemos “organización” en su sentido tradicional: "Una organización es un grupo social compuesto por personas, tareas y administración que forman una estructura sistemática de relaciones de interacción, tendientes a producir bienes o servicios o normativas para satisfacer las necesidades de una comunidad dentro de un entorno, y así poder lograr el propósito distintivo que es su misión”. (en https://es.wikipedia.org/wiki/Organizaci\%0C3\%B3n)
} 
$\mathrm{Al}$ interferir en forma negativa con los elementos requeridos para lograr una adecuada movilización de conocimientos en la organización de la sociedad (Leitsner, 2010), las estructuras universitarias alejan y autoexcluyen a la universidad pública venezolana, y dadas nuestras culturas relacionadas e interdependientes, probablemente a la universidad pública latinoamericana, de esa sociedad que la sostiene y la demanda. Esa sociedad exige un nuevo paradigma que facilite la movilización (detección, generación, transmisión y utilización) de conocimiento para así contribuir eficientemente a la definición de políticas públicas en su seno o en los espacios pertinentes.

Un camino para lograr la aparición del nuevo paradigma universitario que privilegie la movilización de conocimiento lo ofrecen las tecnologías informáticas y las teorías que han ido surgiendo tanto desde la psicología como de la educación con relación al conectivismo de Siemens (2004) y Downes (2011), y que tienen como antecedente la zona de desarrollo próximo de Vygotsky y el constructivismo.

La organización universitaria debería adquirir destrezas conectivistas y aplicarlas orgánicamente para incrementar la eficiencia requerida para el procesamiento del conocimiento disponible y el nuevo a ser creado por los puntos de decisión. Se convierte así la movilización de conocimientos desde la universidad en un problema de la organización universitaria, que no de los profesores o del personal administrativo.

Proponemos iniciar los esfuerzos tendientes a alterar positivamente este estado de cosas, a modo de experimento, a través de proyectos colaborativos a desarrollar entre los programas doctorales en educación, administración y ciencias sociales. En principio los programas doctorales son particularmente adecuados a esta iniciativa por ser momentos donde los docentes, al desdoblarse como candidatos doctorales, permanecen por tiempos relativamente prolongados realizando investigaciones y estudiando metodologías 
que ayudan a llegar a generalizaciones o demostrar hipótesis de trabajo que puedan incrementar la comprensión de una situación dada. Por otra parte, la disciplina de los estudios doctorales y la metodología de tutores y jurados internos y externos a las instituciones podrían favorecer el desarrollo de las "culturas colaborativas" requeridas para la movilización eficiente de conocimiento en nuestro medio.

Una experiencia alineada con esta visión se ha venido desarrollando desde las iniciativas de los Cursos Abiertos Masivos en Línea (CAMEL), en particular los de "Investigaciones Colaborativas en Ciencias Sociales" y en el "Seminario de Investigación sobre la Movilización de Conocimientos entre la Universidad y la Sociedad" dictados desde Programas Doctorales en universidades de Venezuela. Los resultados parecieran señalar la posibilidad de intentar esta vía para lograr en forma eficiente la colaboración al interior de y entre las universidades públicas, para contribuir más eficientemente con los conocimientos que requiera la sociedad.

\section{Los programas doctorales como mecanismo para el cambio paradigmático}

Los sistemas culturales tienden a perpetuarse, esto ha sido demostrado en muchas oportunidades, tanto en la sociedad en general, por ejemplo en estudios de Willis (1977) que reflejan cómo los hijos de las clases trabajadoras aprenden a comportarse como clase trabajadora y los de Lareau (2003) sobre cómo las características de la familia determinan y marcan la vida de los niños que crecen en esos ambientes, así como en el sistema educativo, analizado por Bourdieu y Passeron (1964), de allí las dificultades que enfrenta el intentar cambios en los sistemas socioculturales. La universidad no escaparía a esta realidad, y los programas doctorales reflejarían la cultura organizacional universitaria y el paradigma vigente. Sin embargo, por sus propias características, el subsistema de educación de postgrado, y en particular el de los programas doctorales está en 
situación particularmente adecuada para incrementar la colaboración entre universidad y la sociedad dentro de un nuevo paradigma.

Pero primero debemos revisar lo que persigue el doctorado:

... debe hacerse distinción entre los trabajos especiales de grado generados en los pregrados, especializaciones y maestrías con las tesis doctorales, las últimas tienen por finalidad la creación y construcción de teorías, las primeras tienen un carácter de aplicación y recorrido riguroso del método como garantía del proceso investigativo... Ahora bien, en cuanto al término teoría, se requiere una reivindicación, no entendido como habladuría, sino cómo dar cuenta de la realidad, al respecto, siempre según: el teorizar es el aspecto resaltante de la investigación ya que es el momento en el cual se realiza el acto científico, el hacer ciencia y el generar conocimiento. Por ende, ese es el punto clave de la tesis doctoral, el constructo teórico que emerge como fruto de la investigación. Lo cual recuerda el significado de Ph.D, o doctor pues su tarea es construir y desarrollar filosofía atendiendo al planteamiento de que: la filosofía es un proceso intencionado en la búsqueda de la producción de una episteme y de un conocimiento. (Morales 2011)

Es dentro de esta necesidad de generar teoría explicativa que apunte a la solución de nuestros problemas que Roche hablaba sobre cómo "es indispensable que nos aboquemos a desarrollar un postgrado" (1975b).

Los estudios de postgrado tienen larga trayectoria en Venezuela (ver por ejemplo la revisión realizada por Bifano, 2000) y en el presente están debidamente normados y reglamentados de acuerdo a la Normativa publicada en Gaceta Oficial N. ${ }^{\circ} 37.328$ del martes 20 de noviembre de 2001 . En la misma resaltan una serie de objetivos generales y particulares que entran en conflicto con los requerimientos específicos que se le imponen a los programas y tesis a nivel de maestría y doctorado (ver tabla). 


\section{Disonancias cognoscitivas en la normativa general de los estudios de postgrado para las universidades e institutos debidamente autorizados por el Consejo Nacional de Universidades}

\section{Gaceta Oficial N. ${ }^{\circ} 37.328$ del martes 20 de noviembre de 2001}

\begin{tabular}{|c|c|c|}
\hline De los Objetivos & $\begin{array}{l}\text { De los Programas de } \\
\text { Maestría }\end{array}$ & $\begin{array}{l}\text { De los Programas } \\
\text { Doctorales }\end{array}$ \\
\hline $\begin{array}{l}\text { Art. 1. Se entiende por } \\
\text { estudios de postgrado los } \\
\text { dirigidos a... egresados... } \\
\text { comprometidos con el } \\
\text { desarrollo integral del país. } \\
\text { Art. 2. Los Programas de } \\
\text { Postgrados están dirigidos a } \\
\text { fortalecer y mejorar la perti- } \\
\text { nencia social, académica, } \\
\text { política, económica y ética... }\end{array}$ & $\begin{array}{l}\text { Los estudios de maestría } \\
\text { (Art. 23) "comprenderán un } \\
\text { conjunto de asignaturas y de } \\
\text { otras actividades organizadas } \\
\text { en un área específica de } \\
\text { conocimiento, destinadas al } \\
\text { análisis profundo y sistema- } \\
\text { tizado de la misma y a la } \\
\text { formación metodológica } \\
\text { para la investigación". }\end{array}$ & $\begin{array}{l}\text { Los estudios doctorales } \\
\text { (Art. 26) "tienen por finali- } \\
\text { dad la capacitación para la } \\
\text { realización de un trabajo de } \\
\text { investigación original que } \\
\text { constituya un aporte signifi- } \\
\text { cativo al acervo del conoci- } \\
\text { miento de un área específica } \\
\text { del saber". }\end{array}$ \\
\hline $\begin{array}{l}\text { Art. 4. Los estudios de } \\
\text { postgrado tienen como } \\
\text { finalidad fundamental: } \\
\text { a) estimular la creación y } \\
\text { producción intelectual } \\
\text { como expresión del trabajo } \\
\text { y del estudio. } \\
\text { b) formar recursos humanos } \\
\text { altamente especializados y } \\
\text { promover la investigación } \\
\text { para responder a las exigen- } \\
\text { cias del desarrollo social, } \\
\text { económico, político y cultural } \\
\text { del entorno y a la demanda } \\
\text { social en campos específicos } \\
\text { del conocimiento y del } \\
\text { ejercicio profesional. } \\
\text { c) desarrollar la difusión } \\
\text { cultural, el servicio, la inte- } \\
\text { gración y la interacción con } \\
\text { la sociedad. } \\
\text { d) integrar la extensión } \\
\text { como un proceso de inter- } \\
\text { acción que los actores de la } \\
\text { educación de postgrado } \\
\text { realizan en un entorno } \\
\text { social para aprender de él, } \\
\text { comprenderlo y mejorarlo. }\end{array}$ & $\begin{array}{l}\text { El trabajo de grado (Art. 25) } \\
\text { será "un estudio que } \\
\text { demuestre la capacidad } \\
\text { crítica, analítica, constructiva } \\
\text { en un contexto sistémico y } \\
\text { el dominio teórico y meto- } \\
\text { dológico de los diseños de } \\
\text { investigación propios del } \\
\text { área del conocimiento } \\
\text { respectivo". }\end{array}$ & $\begin{array}{l}\text { Se exigirá (Art. 28) una } \\
\text { Tesis Doctoral, la cual } \\
\text { "debe constituir un aporte } \\
\text { original relevante a la ciencia, } \\
\text { la tecnología o las humanida- } \\
\text { des, y reflejar la formación } \\
\text { científica y humanística del } \\
\text { autor." }\end{array}$ \\
\hline
\end{tabular}


Es de hacer notar el énfasis puesto sobre las áreas del conocimiento en los niveles de maestría y doctorado, en lugar del énfasis en promover la investigación para "responder a las exigencias del desarrollo... en un entorno social para aprender de él, comprenderlo y mejorarlo" presente entre las finalidades específicas de los postgrados. Si bien en los artículos donde se definen los grandes objetivos se hace referencia al entorno, cuando se pasa a los aspectos concretos de lo que se busca en una tesis doctoral o de maestría el énfasis cae en "el acervo de conocimiento" en un "área específica del saber", cuando sabemos que los problemas de la sociedad son por su naturaleza complejos y solo susceptibles de soluciones complejas. Esto es consecuencia del énfasis en la investigación per se, existente en un sistema donde en la medida que se profundiza el habitus universitario, dentro del paradigma de la universidad generadora de conocimiento, se incentiva la publicación académica como un fin en sí mismo.

La situación descrita tiene importantes consecuencias a la hora de orientar nuestra atención al problema de la movilización de conocimientos entre la universidad y la sociedad. El sistema de variables (¿valores?) vigente orienta las investigaciones hacia el interior de la organización universitaria. De esta forma difícilmente podrá el conocimiento generado por esas investigaciones ser de interés para la sociedad.

$\mathrm{Al}$ analizar la normativa vigente para postgrados en Venezuela en lo referente a la organización interna de cada universidad para apoyar esos postgrados, se refleja en sus estructuras el compartimento estanco de las organizaciones a las que pertenecen, aislados del resto de la estructura universitaria (no solo la académica) de manera que nacen solicitando más recursos para lograr sus fines, en lugar de buscar los recursos existentes en la propia universidad o en la sociedad, y optimizarlos en función de las metas planteadas. Así, difícilmente podrán tener los doctorandos las destrezas requeridas para movilizar conocimiento conjuntamente con la sociedad a la cual se deben, o lograr con la ejecución de sus tesis doctorales 
avanzar el conocimiento que la sociedad requiere. Adicionalmente, se pierde la oportunidad de capacitar adecuadamente a los candidatos doctorales, futuros líderes universitarios, en aspectos relativos a la gerencia de las instituciones educativas. Para ello es necesario desarrollar competencias adicionales, sobre todo en el caso de doctorandos en gerencia o educación, orientadas a manejar adecuadamente las estructuras universitarias.

Esta situación de disonancia cognoscitiva se hace patente al analizar lo relativo a la cooperación entre programas de postgrado, de acuerdo a la Normativa Oficial ya citada, que menciona los Órganos Asesores en Materia de Postgrado diciendo que el Núcleo de Autoridades de Postgrado actuará (Art. 5) ... como instancia asesora... en materia de ... coordinación de postgrados en el contexto nacional...; y tiene como atribuciones las siguientes; (Art. 7)... c) Promover los mecanismos y procedimientos de vinculación e integración ...;i) Promover el establecimiento de convenios interinstitucionales y alianzas estratégicas dirigidos a optimizar los recursos de investigación y postgrado.

Sin embargo, en contraste con estas importantes aspiraciones, en las Disposiciones Comunes (Art. 34) tan solo se menciona que “...los estudios correspondientes podrán ser de tipo interinstitucional (integrados)"; y, luego en los artículos 46 y 47. Por la importancia para la discusión los citamos in extenso:

Art. 46. Los organismos de coordinación de estudios de postgrado deben promover convenios entre las Instituciones de educación superior del país y del exterior para fomentar y acordar programas conjuntos en los que se aprovechen las experiencias adquiridas y se eviten las innecesarias duplicaciones.

Art. 47. Las instituciones extranjeras interesadas en desarrollar actividades postgrado en Venezuela, deberán antes del inicio de sus actividades, obtener la correspondiente autorización para la creación y funcionamiento del programa, dentro de lo establecido en los artículos 14 y 15 de la presente normativa. 
Muy alejados de la definición de políticas públicas que permitiese establecer criterios para cumplir con los grandes objetivos delineados al establecer la naturaleza y fines de los estudios de Postgrado.

Si a lo anterior añadimos el hecho de la baja conectividad de la educación en Latinoamérica, a la cual no escapan las universidades en general y los postgrados en particular, veremos que están dadas las condiciones para aislar o al menos alejar a nuestros candidatos doctorales de los problemas de la sociedad que los rodea.

Las consecuencias de esta situación ya son conocidas:

Dos nuevos estudios confirman algo que todos sospechamos desde hace tiempo: las empresas latinoamericanas no pueden competir eficazmente en la economía mundial porque los sistemas educativos de sus países están totalmente desconectados de la realidad. El Reporte Global sobre la Tecnología de la Información 2012, un documento de 442 páginas del Foro Económico Mundial y de la escuela de negocios INSEAD, sitúa a casi todos los países latinoamericanos muy por detrás de los países más conectados tecnológicamente del mundo en su ranking principal de conectividad... Pese al hecho de que recientemente Brasil superó a Gran Bretaña como la sexta economía mundial, los países latinoamericanos que figuran mejor posicionados en el índice son Barbados (35), Chile (39) y Uruguay (44). Ocupando una posición más baja en la lista se encuentran Brasil (65), Colombia (73), México (76), República Dominicana (87), Argentina (92), Perú (106) y Venezuela (107)... El pobre desempeño de Latinoamérica en el ranking se debe "a una débil base de capacitación de la población a causa de mediocres sistemas educativos que obstaculizan la capacidad de la sociedad para hacer un uso efectivo de las tecnologías", así como a la poca inversión en infraestructura de internet de banda ancha, y a climas empresariales desfavorables, según el informe. Andrés Oppenheimer (2012) 
Simultáneamente con esta necesaria inserción de la conectividad, consideramos importante intentar ejercicios de gestión de cambio organizacional a nivel de la gerencia universitaria en nuestros estudios doctorales que persigan y logren alinear los intereses del entorno social con los de la organización universitaria, dirigidos sobre todo a mejorar la movilización de conocimientos entre la universidad y la sociedad, situación que comenzamos a observar, al menos en lo referente al acervo bibliográfico de nuestras universidades (Dávila Quintero et al., 2006).

Una de las herramientas más eficientes para la generación de conocimientos estaría representada por los alumnos cursantes en los doctorados, quienes, con el requisito de sus tesis doctorales, y formando parte de redes abocadas a la comprensión de problemas de nuestras sociedades podrían marcar la diferencia en los procesos por venir. El hecho de que los futuros doctores no solo influyen desde ya la docencia que ejercen en sus cátedras universitarias, sino que eventualmente serán los líderes de esas instituciones, da especial significado a lo que debería ser su preparación en el área de la gestión universitaria.

Este tipo de iniciativas avanzará más rápidamente si se da en el contexto latinoamericano, en lugar de en los espacios nacionales. Un primer paso representado por la iniciativa de generar una base de datos de doctorados en las ciencias sociales, educativas y gerenciales como ejemplo de uso de tecnología para la cooperación ya es posible con la acumulación de datos existentes en el Consejo Latinoamericano de Ciencias Sociales, CLACSO.

Por ejemplo, el CLACSO pudiese ayudar a estimular la inserción transversal de la conectividad (Ordóñez, 2011) en el currículo de los doctorados de manera de propiciar la generación de una cultura colaborativa que nos facilitase resolver los "cuellos de botella" que en la actualidad dificultan esta incorporación acelerada del conectivismo a nuestro mundo académico (Ordóñez, 2012) y por ende a las estrategias para adelantar investigaciones colaborativas de mayor pertinencia social. 
Se puede concluir esta sección con los tres llamados de Devés Valdés (2006):

... El primero está particularmente destinado a los estudiantes universitarios: Les invito a pensar más allá del propio país, a desarrollar un interés por América Latina y por coordinarse con estudiantes de otras universidades de la región, lo que pasa por cierto, por entregar buena cantidad de cursos en la universidad sobre la región.

El segundo llamado apunta a fomentar el estudio del pensamiento latinoamericano. Se trata de una inmensa cantera poco explotada. Creo que es una buena oportunidad para... algo así como una cátedra o departamento o grupo de estudios sobre el pensamiento latinoamericano... Producir la convergencia de estas personas a partir de alguna forma de institucionalidad, parece importante para potenciar el desarrollo de nuestro pensamiento.

El tercero es invitarles a pensar en esto de una Internacional del Conocimiento que nos permita a intelectuales, universitarios y a todos los profesionales del conocimiento asumir el necesario liderazgo en una América Latina que no debe continuar con la vergüenza de permanecer como agua estancada, alimentando podredumbre. Pienso que esta es la mejor manera de aprovechar la iniciativa de la sociedad civil intelectual en orden a articularse para liderar una tarea insustituible que es mover y remover a América Latina".

Los programas doctorales resultan en nuestra opinión los lugares más indicados para iniciar este tipo de procesos preconizados por Devés Valdés, a través de la generación de estudios colaborativos.

Esta generación de estudios pertinentes a nuestra realidad es particularmente urgente en lo social. Para nadie es una sorpresa que el mundo latinoamericano sufre en forma endémica de una serie de problemas sociales. Desde la pobreza, pasando por el autoritarismo, hasta la dificultad para formar ciudadanos, son una serie 
de situaciones que requieren cada vez más de investigación si es que pretendemos generar suficiente información que pueda ser utilizada a la hora de definir políticas públicas para resolverlas. Pero por su misma naturaleza de problemas complejos solo pueden ser analizadas en forma compleja; es aquí donde entra a jugar un papel la estrategia de adelantar investigaciones colaborativas que encuentran en los programas doctorales el terreno abonado para su adecuada instauración y desarrollo.

Acopiar y procesar la información necesaria para poder apenas imaginar la generación de modelos que permitan aproximaciones racionales a su solución no es tarea que pueda ser emprendida por una sola institución, menos aún por un equipo aislado de investigación.

El analizar situaciones en forma simultánea para estudiar cómo diferentes variables interactúan para generar situaciones problemáticas es una labor ciclópea, por costosa y compleja, que solo puede ser intentada cuando diferentes equipos de investigadores, con acceso a diferentes medios ambientes, comparten protocolos y suman sus inteligencias para tratar de profundizar en esos conocimientos necesarios para la toma de decisiones.

Precisamente, una de los problemas que los sociólogos todavía tienen que estudiar en nuestro medio es el porqué de nuestra dificultad a la hora de interactuar colaborativamente, de forma horizontal, para generar equipos eficientes en capacidad de emprender tareas complejas y llevarlas a término.

Esa es precisamente una de las trabas que tenemos a la hora de generar investigaciones colaborativas entre investigadores ubicados en diferentes países, diferentes laboratorios, diferentes disciplinas, pero alineados todos por ese espíritu que nos lleve a compartir ideas, protocolos, resultados, sueños en una suma espléndida que nos permita asesorar a los decisores desde la solidez que brinda el conocimiento generado por el método científico. 
Esa es la ambiciosa meta que nos hemos planteado al iniciar el esfuerzo de generar Cursos Abiertos Masivos En Línea (CAMEL) en temas como Investigaciones Colaborativas en Ciencias Sociales y Movilización de Conocimientos entre la Universidad y la Sociedad, los cuales describiremos en la próxima sección.

\section{Los cursos abiertos masivos en línea realizados en alianza estratégica con programas doctorales en Venezuela}

CAMEL es el acrónimo de Curso Abierto Masivo En Línea, término originalmente acuñado en inglés como MOOC (Massive Open Online Course), y que surge como consecuencia de los experimentos derivados del paradigma de conocimiento conectivista desarrollado por los ya mencionados George Siemens y Stephen Downes en Canadá. El CAMEL busca facilitar el acceso de los ciudadanos a información de calidad, poniendo a disposición de quienes lo quieran un cúmulo de conocimientos desarrollados en línea y en red $^{2}$. Más allá de los intereses de investigación, del aprendizaje de cómo realizarlos y de cuáles son sus resultados desde el punto de visto académico, creemos que los objetivos fundamentales de los CAMEL deben estar orientados a facilitar la divulgación de información en la población general, una forma de aprendizaje emergente (Williams et al., 2012) que creemos resulta sumamente apropiada para el proceso de intervención masiva que planteamos para nuestros postgrados.

Los CAMEL están particularmente bien adaptados para poner información abundante y de calidad (esto último gracias a la generación de los Ambientes Personales de Aprendizaje, APA) a la disposición de los participantes en el proceso. Esto requiere de decisiones en las políticas públicas a ser implantadas, de cambios

2 Ver el siguiente enlace: https://www.youtube.com/watch? ${ }_{\mathrm{v}}=\_$vNWI2Ta0Kk, recuperado el 13 de abril de 2014 . 
en las prioridades dadas a la formación de los docentes, de toda una reestructuración mental del para qué y cómo necesitamos dar educación a nuestros pueblos; tenemos una manera revolucionaria a nuestro alcance para llevar información de calidad a vastos sectores que hasta ahora sencillamente no la habían recibido, pero debemos iniciar el proceso desde las universidades y en particular desde los posgrados.

El solo pensar en los ahorros de costos que se pueden conseguir debería ser suficientes para encontrar la energía de activación necesaria para poner en marcha todo un proceso de desarrollo de experticias de aplicación de CAMEL a problemas concretos dentro del contexto de los programas doctorales que, por definición, reúnen a profesionales capaces y motivados a la investigación por períodos de tiempo relativamente prolongados.

Sin embargo, en nuestro medio no es sencillo insertar estrategias conectivistas en instituciones académicas de tradición. Algunas de las áreas problemáticas se describen a continuación:

1. Contenido de los materiales educativos. Lo digital acarrea una serie de problemas que deben ser manejados por los docentes. La posibilidad de utilizar medios visuales, voz, gráficas y la posible interacción de los participantes entre ellos y con el docente, obliga a considerar la adaptación de los materiales previamente utilizados para la docencia tradicional presencialexpositiva. Un ejemplo concreto lo presenta el problema de la duración de las clases virtuales, más de 20 minutos de exposición no son recomendables, de acuerdo a la experiencia. Otro ejemplo, el formato de presentaciones en láminas (del tipo representado por el programa PowerPoint) y la ya famosa recomendación 10-20-303.

3 Popularizado en los últimos tiempos, se refiere a no utilizar más de 10 diapositivas o láminas en un tiempo máximo de 20 minutos y utilizando letras de tamaño 30 para evitar presentar textos a la audiencia (ver por ejemplo el siguiente enlace: http://cosas sencillas.wordpress.com/2007/01/30/presentaciones-102030/ recuperado el 13 de abril de 2014). 
2. Las plataformas donde se montan los cursos. Los cursos se montan en plataformas digitales, su dominio y requerimientos técnicos varían, las hay desde muy completas (y complejas) como la Plataforma MOODLE o las desarrolladas por COURSERA, hasta las ad-hoc, como pueden ser combinaciones de grupos de reunión tipo YAHOO GROUPS (para agrupar a los cursantes y como aula virtual del curso), WiZiQ o YouTube (para el dictado de clases con láminas, sonido y pizarrón digital), SKYPE (para conversar y apoyar en aula compartida) y Google Drive (para compartir esfuerzos en la elaboración de documentos), en cualquier caso, la administración simultánea de todas estas plataformas requiere un nivel de experticia que deberá ser dominado por el docente que ya conoce y presenta los contenidos educativos.

3. Las experticias en la utilización de las herramientas informáticas. El dominio en la utilización de herramientas informáticas, tanto para obtener información como para procesarla individual o grupalmente, varía mucho en cualquier población en estos momentos. En cada programa podemos encontrar desde docentes que conocen y desarrollan instrumentos, hasta docentes con amplia experticia en su campo de conocimientos pero que se niegan a entrar en el mundo virtual. Igual situación encontramos en los estudiantes, desde aquellos con amplio dominio hasta los que tienen un conocimiento apenas superficial de algunas herramientas de comunicación. Todo esto lleva a problemas de curvas de aprendizaje diferentes a la hora de un curso particular. Encontraremos individuos que pueden poner manos a la obra de inmediato, mientras que habrá otros que primero deberán dominar las herramientas para solo después iniciar procesos de aprendizaje de la materia propiamente dicha. Los mayores problemas se encuentran cuando difieren en forma cruzada las experticias de docentes y cursantes, siendo el peor caso aquel donde los estudiantes dominan herramientas que los docentes no conocen, su solución dependerá en mucho de lo tratado en el siguiente punto. 
4. Aspectos organizativo-culturales. Las tres áreas problemáticas revisadas hasta ahora acarrean naturalmente conflictos que se deberán ir resolviendo a lo largo del curso, dentro de las ya mencionadas curvas de aprendizaje. No es lo mismo iniciar un curso digital por primera vez en una institución que se inicia en estos menesteres que dictar por enésima vez ese mismo curso probado donde el personal de apoyo y los docentes ya se conocen. Así, montar cursos en ambientes digitales, utilizar adecuadamente las plataformas, tanto por profesores como por estudiantes, e interactuar en condiciones disímiles de experticia informática, pueden generar tensiones y conflictos de mayor o menor grado dependiendo de la cultura de la sociedad en que los procesos se producen y las condiciones particulares de la organización en cuyo seno se realizan. En particular nos referimos a variables como el manejo de la autoridad; las formas de obtener y procesar información; socialización de conflictos; culturas colaborativas; y mayor o menor resistencia al cambio. Todos estos factores influyen en la forma en que se resuelvan (o no) los conflictos que se vayan presentando.

5. Los equipamientos requeridos. Tanto a nivel de máquinas y herramientas digitales (computadoras, servidores, teléfonos móviles, software y demás), como de infraestructura nacional o local en telecomunicaciones y teleinformática, estos factores van a influir decisivamente en la posibilidad real de iniciar procesos de virtualización de la educación por una institución, tanto a la hora de dictar cursos presenciales con el apoyo de plataformas informáticas tipo MOODLE, como en cursos semipresenciales o aquellos que se dictan totalmente a distancia. Estos factores escapan al control de las organizaciones educativas y deberán ser considerados en cada caso particular.

Ver figura: Problemática en la inserción de la conectividad. 


\section{Figura $\mathbf{N}^{\circ} 1$}

Problemática en la inserción de la conectividad

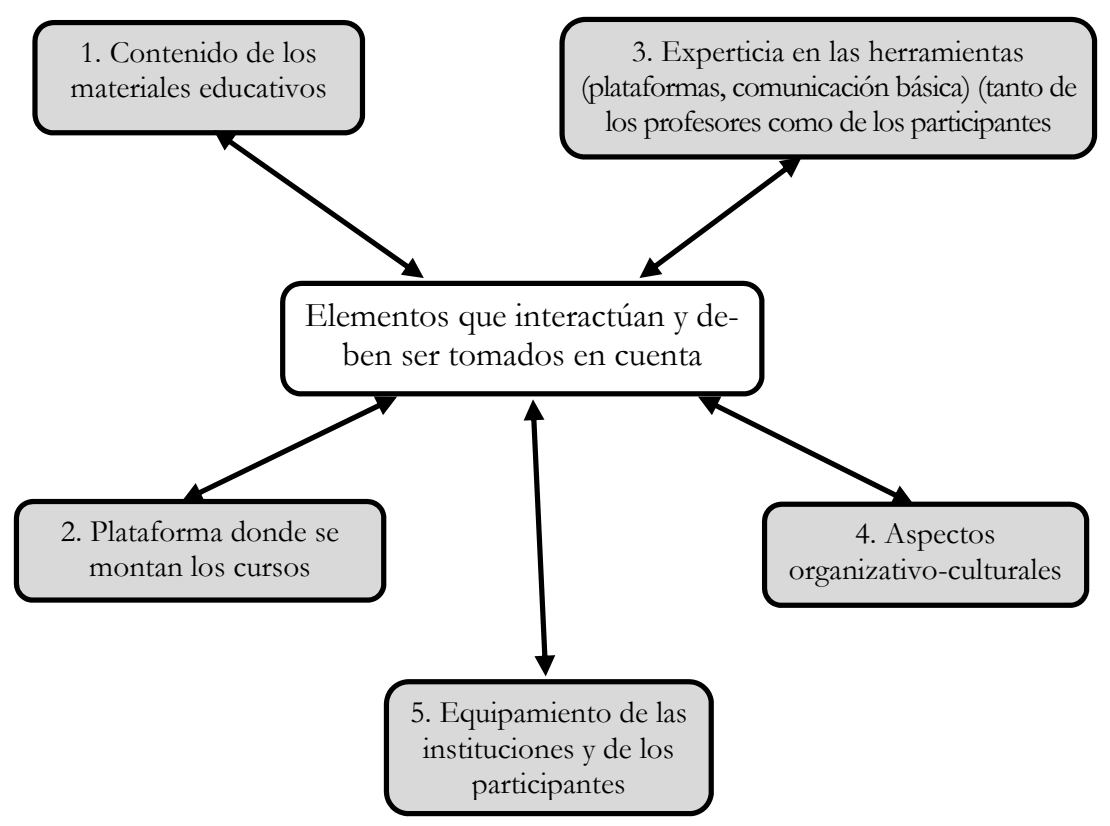

Elaboración propia.

\section{El mínimo común denominador}

De partida, una institución dedicada a dictar cursos y programas profesionales para adultos decide desarrollar un nuevo paradigma educativo, basado fundamentalmente en la adopción de la semipresencialidad, con base en las conocidas ventajas que brinda este tipo de instrucción, en especial que los estudiantes aprenden más con la enseñanza en línea, entre otras cosas, por incorporar el aprendizaje conectivista a sus estrategias de obtención y procesamiento de información. El proyecto adelantado, al pretender facilitar la semipresencialidad, trae como consecuencia, en adición a la consecuente adopción de recursos informáticos, el desarrollo 
de un proceso que permite a los cursantes aprender más y seguir aprendiendo después de tomar los programas de la institución.

Uno de los problemas que encontramos en la actualidad, a la hora de generar cursos abiertos masivos en línea, reside en la heterogeneidad de los participantes que pueden inscribirse en el curso y sus diferentes "niveles de conectividad", es decir, su mayor o menor experticia en la utilización de internet y las herramientas desarrolladas para la web 2.0. Como ya comentamos, en cualquier curso que se inicie, prácticamente de cualquier nivel, tendremos personas con educación formal en áreas de informática educativa, simultáneamente con otras que apenas se inician en el uso de internet. Esto, unido al hecho de que el sistema educativo en América Latina, por lo general, todavía no prepara al estudiante para obtener y procesar información utilizando la web, obliga a dedicar parte importante al inicio de cada nuevo curso a desarrollar un "mínimo común denominador" de "experticia conectiva" en el grupo.

En adición al hecho inmediato de diferentes experticias, nos topamos con las diferentes actitudes en los participantes a la hora de enfrentar el hecho tecnológico. Se pueden conseguir individualidades que conocen poco de internet, lo aceptan y tienen una actitud positiva frente a los nuevos aprendizajes requeridos; por el contrario, se encuentran participantes a quienes el solo hecho de pensar que van a tener que aprender a usar "programas de computación" los paraliza. Igualmente habrá miembros del grupo que creen saber mucho, y por alguna razón no aceptan que deben aprender más.

Todos los ejemplos del párrafo anterior corresponden a diferentes puntos en las curvas de aprendizaje y son susceptibles de encontrarse al inicio de cualquier curso abierto en línea, de manera que la primera recomendación que debe darse a un docente que pretenda generar uno de estos CAMEL, es dedicar las primeras sesiones del curso a generar ese "mínimo común denominador de experticia 
informática" entre los participantes, con el objeto de que el grupo pueda desarrollarse colaborativamente en las etapas intermedias y finales del mismo. Caso contrario las deserciones pueden aumentar en las primeras etapas, tanto en los participantes con menos "conectividad", por sentirse incapaces de cumplir con las exigencias informáticas del CAMEL, como entre los participantes con más experticia en el uso de estas herramientas, por considerar que se pierde mucho tiempo en nimiedades y no se termina de entrar en materia.

En nuestra experiencia, para facilitar la inserción de la conectividad como parte de los programas regulares de una institución hemos encontrado útil el dictado, al inicio de cada programa, de un taller Aprendiendo a Aprender en la WEB, (Taller AAW) 4 de tres horas de duración, seguido de ejercicios no obligatorios a ser desarrollados por los cursantes a lo largo de dos semanas, con apoyo docente virtual. Es de hacer notar que los talleres los hemos dictado en forma semipresencial o totalmente presencial, otra posibilidad la constituye el dictado del Taller AAW aisladamente, no como parte de un Programa más amplio. En realidad, la inserción del conectivismo puede tomar varias alternativas, dependiendo de las características de la cultura organizacional de la institución educativa y del nivel de experticia logrado por los participantes previo al inicio de los mismos; de igual modo, en función de estas variables, pudieran ser consideradas las alternativas de dictar un Taller AAW de pocas horas, o un Curso AAW de varias semanas, así: Las alternativas dependerán, entre otras, de las experiencias acumuladas y del grado de dominio e interés en las herramientas logrado por los mismos docentes responsables de los diferentes módulos abarcados por la institución en sus programas, de lo contrario el esfuerzo será percibido como aislado, tanto por los docentes como por los cursantes. Lo importante en este punto es lograr la excelencia en

4 Taller desarrollado por la Fundación Interconectados. 
La movilización de conocimientos en la sociedad: Un reto para los programas doctorales en la universidad pública latinoamericana

los programas desarrollados por la institución, tanto a nivel del aprendizaje y satisfacción lograda por los participantes, como por la visibilidad y prestigio que logre la organización.

\section{Figura N. ${ }^{\circ} 2$}

\section{Cuándo insertar la conectividad digital en un programa académico}

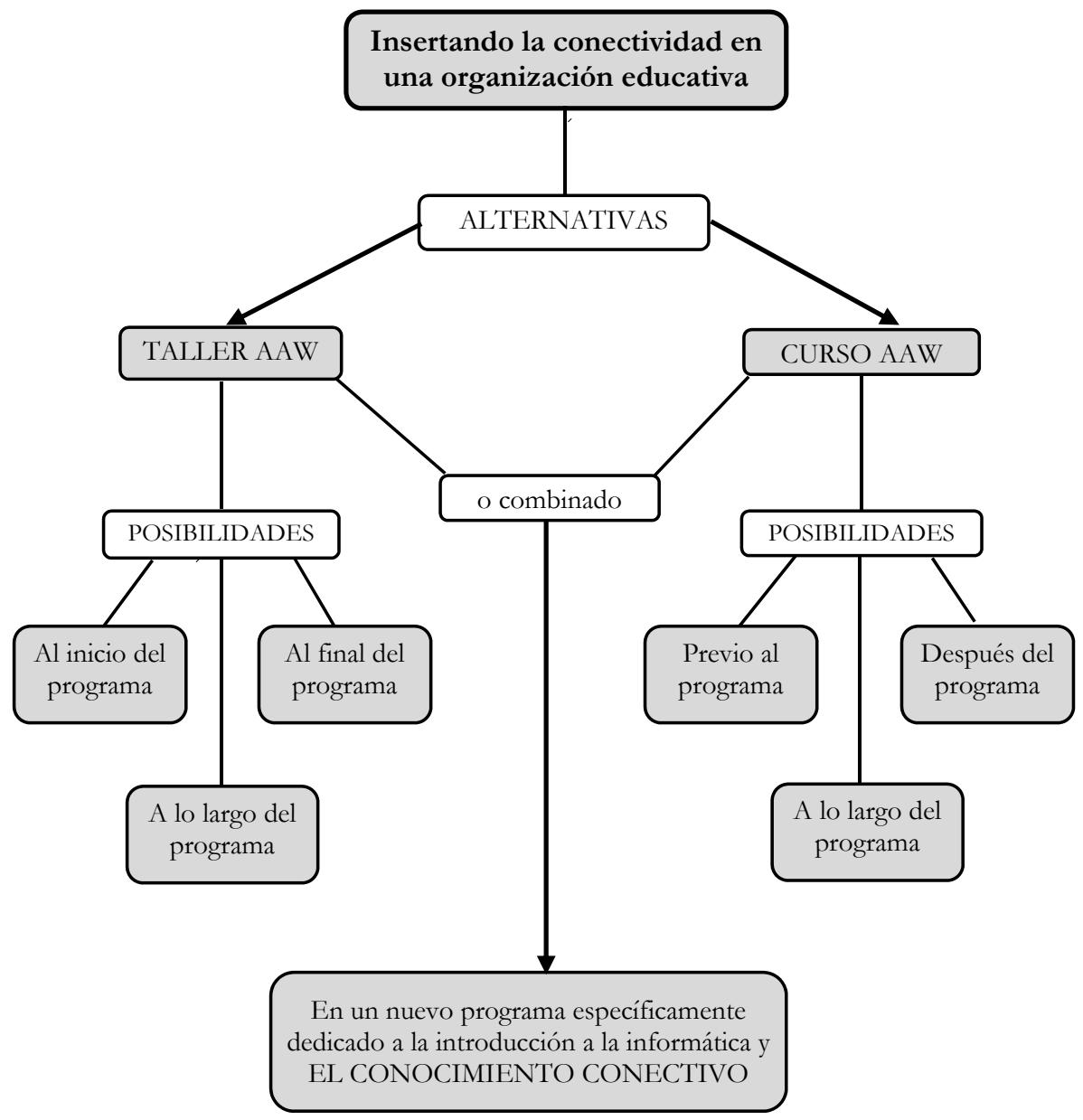

Elaboración propia. 


\section{Las culturas colaborativas}

Para alcanzar máximos resultados en la interacción virtual, se requieren culturas colaborativas que aún no son desarrolladas por nuestro sistema educativo o por nuestra sociedad. Este es un punto que se viene investigando, porque ciertamente las culturas colaborativas se desarrollan en los procesos de interacción en internet y a su vez los aprendizajes se potencian; sin embargo, en nuestra cultura autoritaria el espacio educativo se maneja desde el profesor hacia los alumnos en forma distributiva, en lugar de hacerlo en red y por ende colaborativamente. Igualmente, la forma como se maneja el conocimiento en las luchas propias de "la violencia simbólica" universitaria dificultan la colaboración para el aprendizaje. El comprender estas barreras para el aprendizaje eficiente, y dominarlas se convierte así en otro ejercicio obligado en cada curso que se dicta.

También, en parte encontramos el fenómeno de la "atenuación"5, característico de esta cultura y que dificulta la confrontación franca de ideas entre individuos que no se conocen lo suficiente. En su libro La variable independiente, Alberto Rial (1997) escribe:

Venezuela es un caso de bajo capital social. El sistema de valores, centrado en la familia, desconfía del ciudadano común y obstaculiza los propósitos conjuntos, sean estos una empresa comercial, un gremio o una asociación sin fines de lucro: la desconfianza cultural, en esencia, se traduce en una capacidad limitada de organización.

Por su parte, Granell de Aldaz, Garaway y Malpica, en Éxito gerencial y cultura (1997) señalan:

5 La atenuación es una estrategia que se explica dentro de las actividades argumentativas dirigidas a lograr el acuerdo o aceptación del otro (incluida la aceptación social). Consiste en una operación de minimización de lo dicho y del decir: de lo dicho, en tanto se hace borroso o menos explícito lo enunciado, y del decir, en tanto suavizar la fuerza de mis acciones e intenciones. 
Nos gusta trabajar en grupo, pero ello no significa que trabajemos en equipo y que cada quién asuma su rol dentro de él. En ambos casos la consecuencia es la misma, equipos poco eficientes que no logran alcanzar las metas para las que fueron creados. Creemos que, al menos parte del problema en los ejemplos mencionados, reside en cómo manejamos la comunicación entre los miembros de una organización en nuestro medio.

En nuestra opinión esta estrategia (la atenuación) es adecuada a la hora de mantener las relaciones en el equipo, por ejemplo de la "autoimagen" del docente en academia, pero resultan contraproducentes a la hora de luchar para alcanzar las metas en ambientes virtuales ya que inhibe la participación. Desafortunadamente, internet no facilita la comunicación verbal, dificultando así la utilización de mecanismos de atenuación que facilitan el poder dar a conocer nuestras deficiencias y expectativas al grupo, el lograr la confianza que nos facilite el solicitar apoyo para entender lo que se persigue, o dominar las herramientas requeridas para lograrlo. Todo lo anterior trae como consecuencia el fracaso de muchas iniciativas que, de prosperar, podrían tener consecuencias benéficas para la sociedad.

Solo al final, una vez atacados los problemas hasta aquí mencionados, puede el docente abordar el tema del curso propiamente dicho, donde es un experto, pero que podría tener poco impacto en los alumnos si no se conocen los problemas (reales y potenciales) de estos CAMEL conectivistas.

\section{La experiencia}

Luego de haber dictado varios CAMEL dirigidos a público general ("Participación y Democracia", y "Emprendimientos Cooperativos en Conectividad", entre otros), iniciamos el proceso de promover CAMEL para programas doctorales de universidades públicas 
venezolanas. En alianza estratégica con el Programa de Doctorado en Ciencias Sociales y Humanidades de la Universidad Simón Bolívar (DCSH-USB) se dictó el CAMEL "Investigaciones colaborativas en ciencias sociales" y en varias oportunidades posteriores, bajo el título general de "Movilización de conocimiento entre la universidad y la sociedad", en alianza estratégica con el Programa de Doctorado en Educación de la Facultad de Educación de la Universidad de Carabobo (FaCE-UC) y con el Doctorado en Ciencias Administrativas y Gerenciales de la Facultad de Ciencias Económicas y Sociales de la misma universidad.

En el primer curso, Investigaciones Colaborativas, con el objetivo explícito de que los participantes: "conozcan, comprendan y adelanten estudios colaborativos en su área de interés dentro de las ciencias sociales, bajo una perspectiva interdisciplinaria", el CAMEL estuvo abierto a todas las personas que expresaron interés, sin distingos de carrera o grado académico alcanzado (además, como es usual en los CAMEL, fue totalmente gratuito), aunque su núcleo inicial lo constituyeron cursantes regulares del Programa DCSH-USB.

Dictado a lo largo de 12 semanas; estuvo dividido en tres unidades básicas:

1. Investigaciones Colaborativas en las Ciencias Sociales en el Mundo.

2. Investigación Colaborativa en Ciencias Sociales en América Latina.

3. Investigación colaborativa entre diferentes programas doctorales.

Sin embargo, desde el inicio se realizaron ejercicios para estructurar proyectos de investigación colaborativa, que continuaron a todo lo largo del mismo, y constituyeron gran parte de la evaluación final del CAMEL. El objetivo no es otro que contribuir a la realización de los propios ejercicios individuales de tesis, tendentes a 
completar los requerimientos de investigación a los participantes en la mayoría de los programas doctorales en nuestro medio.

Los otros CAMEL, "Movilización de Conocimiento entre la Universidad y la Sociedad", se insertó como asignatura formal de los dos programas doctorales de la Universidad de Carabobo, Venezuela, dentro de las programaciones regulares de Seminarios de Investigación, pero adicionalmente pueden ser seguidos por cualquier persona interesada, aspecto inherente a un CAMEL. El propósito de estos últimos CAMEL ha sido que los participantes incorporen nuevas realidades en su pensamiento sobre los problemas culturales de nuestra sociedad, la toma de decisiones de políticas públicas, y el nuevo paradigma que deberá surgir para orientar la acción de las organizaciones educativas.

El seminario se concreta en tres bloques temáticos:

1. Herramientas de informática educativa para la acumulación y socialización de información.

2. Estrategias para la investigación colaborativa en Ciencias Sociales.

3. Investigación sobre "problemas para el trabajo de investigación colaborativo y la movilización de conocimiento en la sociedad latinoamericana".

El primer bloque temático pretende hacer un rápido inventario de diferentes herramientas informáticas que pueden ayudar, por una parte, a obtener información sobre temas específicos, y por la otra a organizar y socializar la información obtenida. Simultáneamente se aspira a lograr el ya mencionado "mínimo común denominador" en experticia informática, que permita a los participantes interactuar eficientemente en proyectos colaborativos mediante el uso de internet y la web. Se utilizan herramientas (algunas ya mencionadas) tales como Grupos Yahoo, blogs, plataforma WiZIO, Skype, Google Drive, Cmaps y se apoya el 
aprendizaje con el también mencionado "Taller Aprendiendo a Aprender en la WEB".

El segundo bloque temático parte de los problemas culturales (en el sentido sociológico del término) presentes a la hora de establecer relaciones colaborativas en la sociedad y las estrategias que se pueden utilizar para resolver los cuellos de botella existentes. Lo anterior se desarrolla alrededor de la generación de un proyecto de investigación "colectivo" (a ser desarrollado en grupos de varios participantes) que sin estar dirigido directamente al proyecto de investigación individual de cada uno de los miembros del equipo, pueda ser útil para sus objetivos finales (individuales) de investigación. La temática general de los proyectos así concebidos gira alrededor del problema general estudiado en el seminario: la movilización de conocimientos entre la universidad y la sociedad.

El tercer bloque temático desarrolla una experiencia de investigación "cualitativa" alrededor del tema central del seminario: detectar cuáles son los obstáculos que se enfrentan a la hora de generar proyectos de investigación (generación de conocimientos) o de su socialización (posibles usuarios interesados en los resultados) en forma colaborativa (participación racional de las partes, no mediada por el azar). Para ello, cada grupo conformado en el bloque temático anterior, intenta ampliar su grupo de investigación (primera parte) y de obtener agentes interesados en financiar, utilizar o incorporar los hallazgos de sus investigaciones (segunda parte). El registro de su experiencia y la diseminación de los resultados es la principal actividad evaluadora del trabajo y calidad lograda por los participantes u puede ser utilizada en la evaluación requerida para la aprobación del seminario. 
La movilización de conocimientos en la sociedad: Un reto para los programas doctorales en la universidad pública latinoamericana

\section{Figura $\mathbf{N} \circ^{\circ} 3$}

Estrategias de búsqueda de colaboradores inter-organizaciones para proyectos de investigación en ciencias sociales

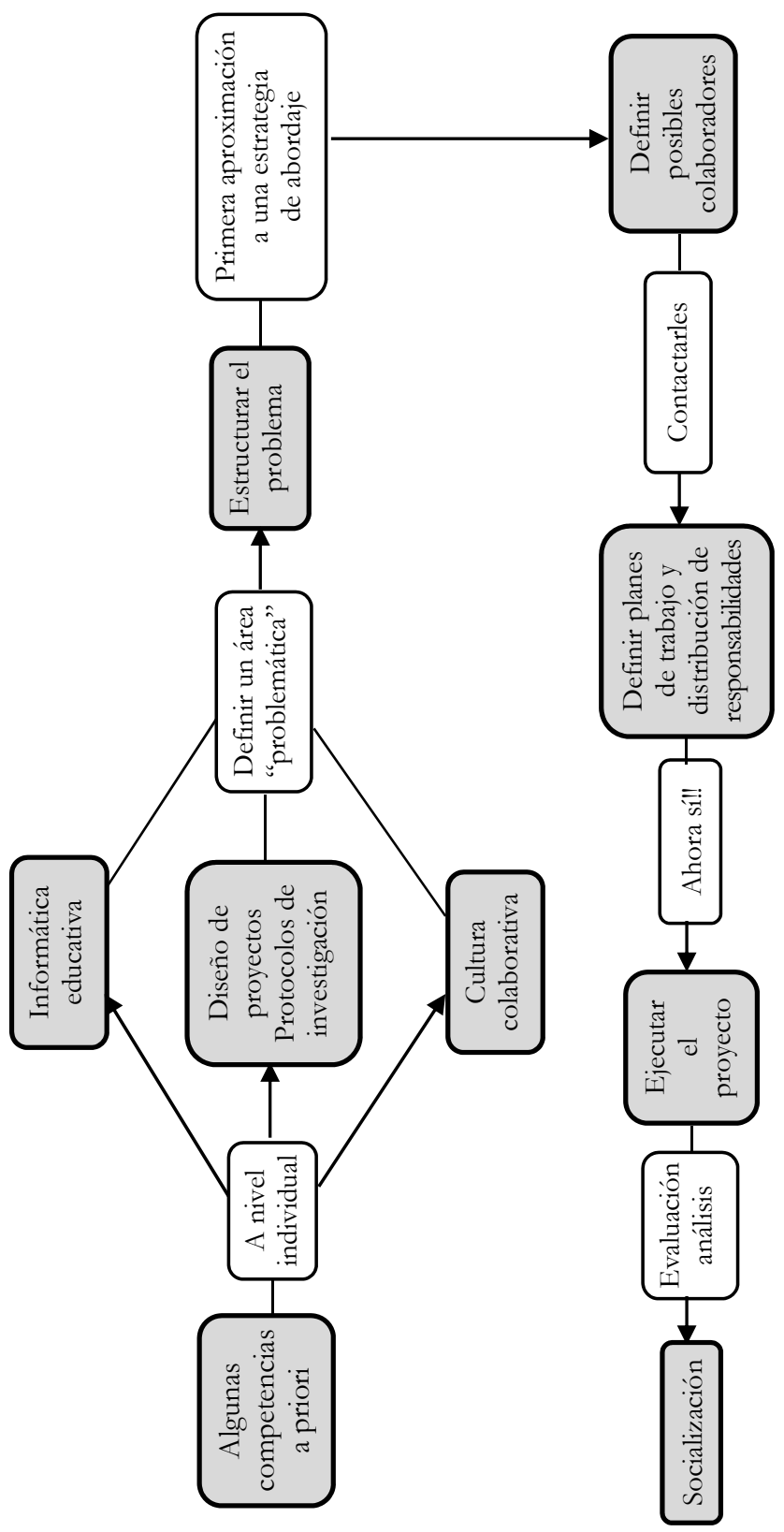

Elaboración propia. 
En la figura vemos una aproximación al análisis del problema. En primer lugar, hay que considerar las competencias que deben poseer los investigadores involucrados. Por lo general, la experticia del profesor universitario en programas doctorales en nuestro medio en tópicos tales como informática educativa, diseño de protocolos de investigación y cultura colaborativa y estrategias sociales para la colaboración no es alta. Si bien podemos desarrollar en lapsos relativamente breves competencias instrumentales en informática y generación de protocolos, la cultura colaborativa choca en mucho con la cultura prevalente en nuestra sociedad autoritaria y motivada al poder, por lo que una aproximación a la solución de este cuello de botella debe pasar por racionalizar el problema y buscar a nivel individual estrategias de solución.

Pero en adición a las competencias individuales necesarias, el investigador interesado en realizar investigaciones sociales colaborativas debe también disponer del tiempo necesario para estructurar adecuadamente el problema en cuestión, conocer a fondo la literatura pertinente para detectar otros posibles investigadores colaboradores-académicos y negociar con estos los planes de trabajo, distribución de responsabilidades y demás aspectos administrativos de todo proyecto, por no hablar del tiempo requerido para detectar, convencer y generar redes de colaboración entre los investigadores.

Aún más, antes de realizar los proyectos de investigación con impacto social, el o los investigadores deberán invertir tiempo y esfuerzos adicionales para generar una estructura social de colaboración en red con autoridades, comunidades, actores importantes y demás, quienes de una u otra manera están involucrados en el desarrollo exitoso de un proyecto de intervención social, o de investigación que lleve a esa posible intervención en aras del mejoramiento de la calidad de vida de una población.

El peor enemigo de todo este proceso es lo que los angloparlantes llaman "wishful thinking" ("hacerse ilusiones" o "buenos deseos") 
y que preferimos llamar en nuestro ámbito latinoamericano "macondismo" (Volek, 2007), el creer que por imaginarnos algo ya lo hemos logrado. Nos topamos permanente con "proyectos de investigación" que se limitan a describir una situación problemática y saltan a concluir que "entonces lo resolvemos", sin estructurar el problema en cuestión, reflexionar sobre los procesos administrativos involucrados o definir los protocolos de investigaciones de campo propiamente dicha.

Contra todo esto deberá luchar en su esfera personal cada investigador universitario antes de salir al mundo a tratar de generar o incorporarse a redes de investigadores interesados en colaborar para solucionar los problemas que presenta su entorno social. Su principal aliado en ello será el conectivismo.

El tipo de resultado que se obtiene en los CAMEL es gratificante, tanto para los participantes como para el facilitador. A título de ejemplo podemos mostrar el resultado obtenido en el de movilización de conocimientos entre la universidad y la sociedad, con más de 30 participantes, donde un total de 15 adelantaron el ejercicio de conformarse en grupos de tres para, con base en sus propuestas preliminares de temas de tesis, generar proyectos de investigación colaborativa.

Los títulos de los cinco proyectos preliminares así generados fueron:

1. Promoción de la Salud Bucal en la Población Infantil.

2. Modelización Matemática como estrategia Didáctica para la enseñanza de la Estadística.

3. Proyecto Canaima Educativa (computadoras para estudiantes de educación primaria).

4. Competencias genéricas de los docentes de Educación Superior.

5. Idioma inglés entre prestadores de servicios turísticos. 
Figura N. ${ }^{\circ} 4$

Investigación integrada como resultado de un CAMEL

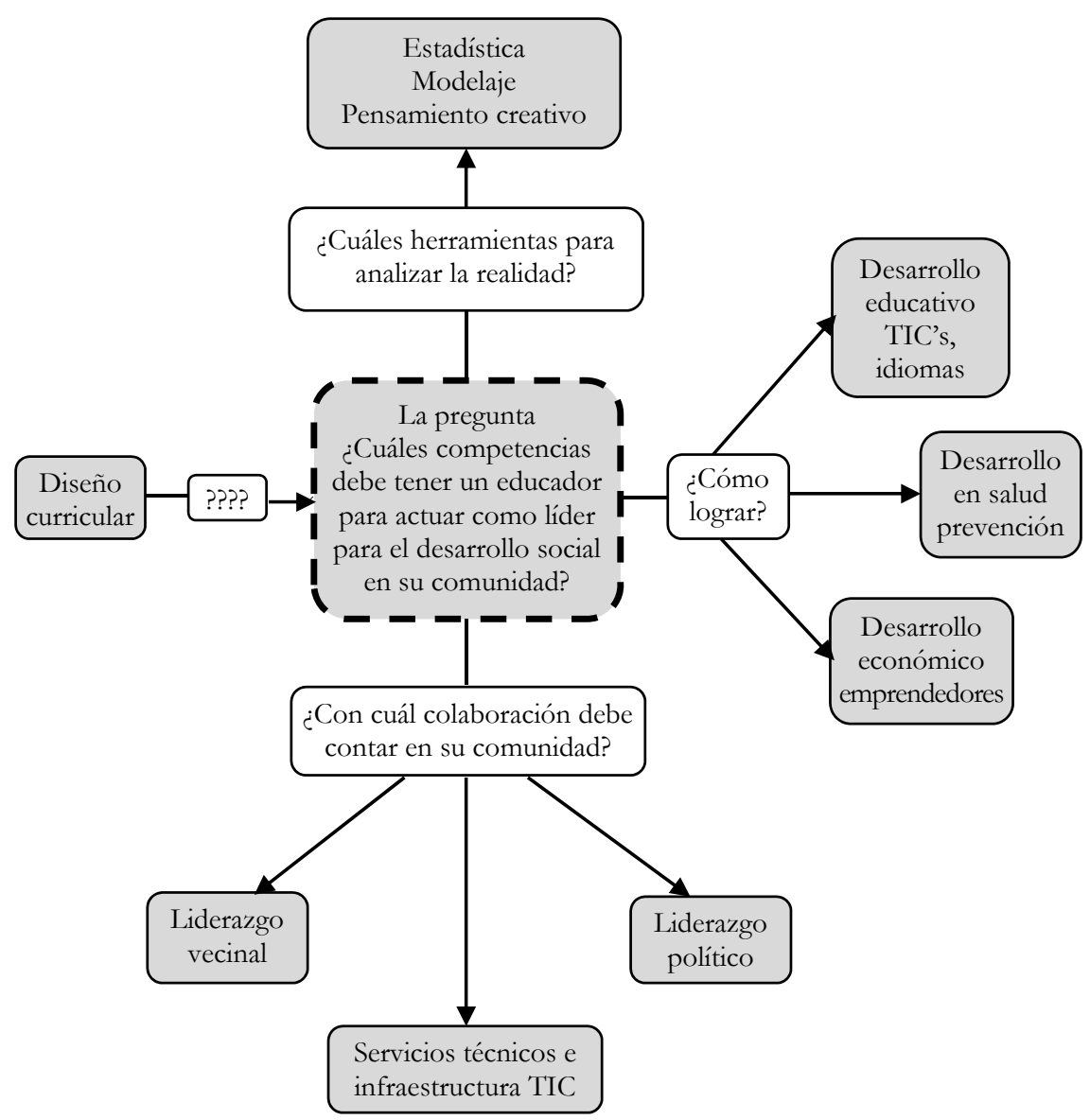

Elaboración propia.

Esto permitió crear una matriz de investigación para generar conocimiento aplicable a comunidades que se nutriera de todos los proyectos y por lo tanto, aportase a los intereses de cada participante. 


\section{La inserción de las investigaciones}

Los investigadores en las ciencias sociales sienten una presión constante por poder relacionar lo que hacen con las necesidades más apremiantes de la sociedad. En otras palabras, demostrar que sus estudios contribuirán en el corto o largo plazo a lograr una mejor calidad de vida para el común de la gente.

Esta situación se puede profundizar en los investigadores de universidades públicas, después de todo su salario está siendo pagado con los impuestos que la colectividad paga al Estado. En los CAMEL que dictamos, en particular aquel sobre Movilización de conocimientos entre la universidad y la sociedad, se le requiere a los participantes que traten de relacionar sus temas de investigación con alguno de los aspectos que influyen en el complejo problema tratado, como una manera de iniciar la conceptualización requerida para abordar las dificultades inherentes a movilizar conocimientos en nuestras sociedades latinoamericanas.

Es decir, se pide racionalizar la importancia de las investigaciones, no en sí mismas, sino ubicadas en el problema de la dinámica social en que está inmerso el investigador y su universidad.

Cómo pudiéramos medir esa inserción: Identificar autores e instituciones mediante el acopio bibliográfico de los proyectos grupales de investigación y analizar la cantidad de vínculos que se pudieran establecer con profesores o alumnos de otros programas doctorales. Igualmente identificar el tipo de organizaciones (públicasprivadas; educativas-sociales; sin fines de lucro-emprendimientos) más dispuestas a colaborar con las universidades para generarutilizar conocimiento. Involucrar estudiantes de pregrado en los procesos de investigación-acción en comunidades aprovechando los programas de Servicio Comunitario a efecto de mostrar formas de intervención, mecanismos de movilización de información y sistematización del uso de las TIC's. 
En conclusión, la conectividad nos ofrece mecanismos para romper patrones culturales que vienen manteniendo alejadas a las universidades públicas del mundo social en su dimensión más amplia, las innovaciones curriculares y las didácticas requeridas están siendo exploradas y son susceptibles de ser dominadas, de manera que vemos un camino para lograr el cambio paradigmático necesario para convertir a la universidad en movilizadora de conocimiento en la sociedad, que no es otro que la de transformar aceleradamente a los cuadros docentes del futuro inmediato mediante la intervención a nivel de los programas doctorales.

La implantación de estos procesos, y su evaluación y ajuste posterior ya está teniendo lugar en nuestro medio, pero es necesario ampliarlo a escala continental para que sus bondades puedan ser apreciadas a la velocidad que son requeridas. Adicionalmente requerimos de apoyos de los avances que se están produciendo en el mundo sobre cómo gerenciar el proceso de reunir, interpretar y comunicar conocimientos para la toma de decisiones.

\section{Recomendaciones para la toma de decisiones}

Si partimos desde los hallazgos más importantes de nuestras experiencias con CAMEL, para desde allí tratar de reconstruir lo que pudiese ser la posibilidad de visualizar un enfoque general para mejorar la eficacia, la eficiencia y la efectividad de la gestión universitaria, debemos recordar que los mismos, tanto en los logros como en las deficiencias detectadas, fueron los siguientes:

1. La posibilidad de generar materias en forma colaborativa entre diferentes programas doctorales, sin necesidad de normativas rígidas, en base a la comunicación entre coordinadores de programas. En nuestro medio es necesaria la interacción personal, base de la confianza social. El capital social debe ser desarrollado. 
2. Alta receptividad de los participantes a las presiones impuestas por las tecnologías, la baja conectividad y el desconocimiento de herramientas. Sí se pueden insertar las tecnologías en nuestros ambientes académicos, aunque se debe estar preparado para la natural resistencia al cambio, que debe contraponerse al sentido de misión que debe conllevar el aprendizaje.

3. Alto nivel de creatividad demostrada en la generación de proyectos colaborativos. Debemos aprovechar al máximo esa cualidad desarrollada en nuestro medio para contrarrestar el impacto negativo de la ausencia de normas claras y/o supeditadas a la cultura autoritaria, que permite a los jefes cambiar las normas de acuerdo a su conveniencia. Nuestra creatividad es nuestra mejor arma y debemos aprovecharla al máximo.

4. Con relación al número de participantes: bajo poder de convocatoria por los canales regulares (convocatoria a otros coordinadores doctorales, web social y demás). Unido al punto 1, la red de capital social será más eficiente en etapas iniciales que los canales formales (inexistentes o no creíbles en base a la experiencia).

5. Con relación al bajo nivel de participación activa entre los que siguen los programas como observadores, un mínimo de opiniones debido, al menos parcialmente, a la ausencia de una cultura colaborativa. Hacer explícito el problema a los participantes desde el inicio de cada actividad y solicitar colaboración activa para resolverlo en una estrategia positiva.

Son los egresados de estos programas doctorales los que eventualmente pueden lograr el paradigma de "La universidad como movilizadora de conocimientos para la sociedad", son estos participantes en programas doctorales los que se encuentran en mejores condiciones para lograr que sus acciones dejen señales que serán percibidas por otros y determinarán próximas conductas. 
Desde este punto dirigimos nuestra mirada final a la universidad. Estamos acostumbrados a percibirlas como centros de excelencia donde se genera y transmite conocimiento socialmente útil. Pero pocas veces nos detenemos a considerar la otra función de la universidades, la de difundir conocimientos a toda la sociedad. La figura $\mathrm{N}{ }^{\circ} 5$ refleja lo que queremos transmitir.

El lector podrá apreciar como en el nuevo esquema asignamos funciones bastante diferentes de las tradicionales a servicios como los de biblioteca y de prensa, puesto que ahora comparten la función de ayudar a satisfacer las demandas de conocimiento que pueda tener el entorno social de la universidad. Una adecuada reingeniería de los sistemas de información y comunicación de la universidad son necesarios para poder cumplir eficientemente estas funciones. Son parte de los nuevos retos de los gerentes de la educación superior y, por lo tanto, es de esperar que entre los participantes en esos programas doctorales se encuentren los futuros gerentes y funcionarios de esas necesarios piezas de la organización universitaria.

Es desde un nuevo tipo de organización, de un nuevo tipo de egresado al más alto nivel académico (los nuevos doctores) y de un nuevo egresado universitario "colonizando" la sociedad, que lograremos restablecer el vínculo perdido entre ambos sistemas, y con ello, la movilización adecuada de los conocimientos requeridos, en el lugar adecuado y en el momento justo. La universidad pública latinoamericana le debe este esfuerzo a esa sociedad que la ha protegido y cultivado a lo largo de todos estos años. 
La movilización de conocimientos en la sociedad: Un reto para los programas doctorales en la universidad pública latinoamericana

Figura N. ${ }^{\circ} 5$

Hacia nuevos diseños organizativos para la universidad movilizadora de conocimiento

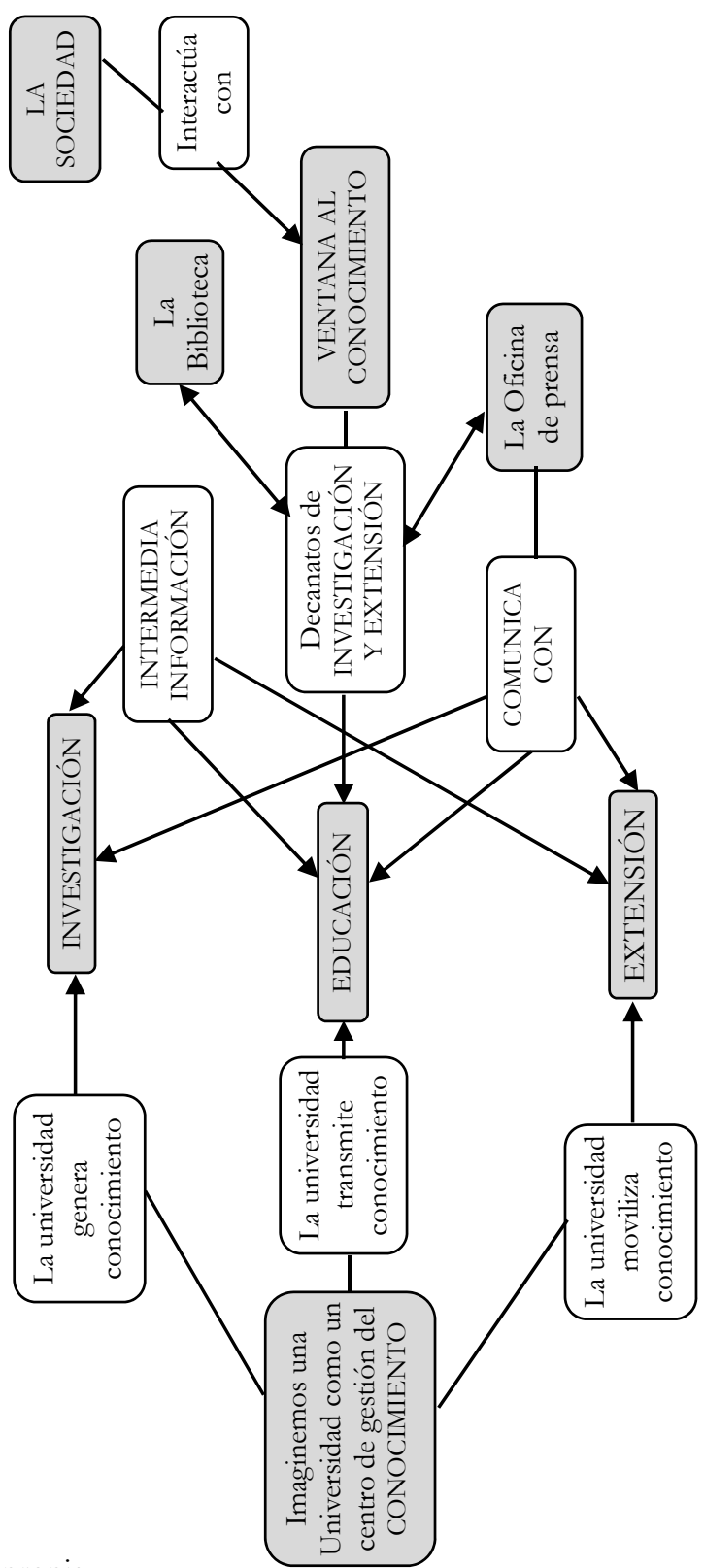

Elaboración propia. 


\section{Bibliografía}

Bifano, C. (2000). Los grados académicos del postgrado. La necesidad de fortalecer la especialización. Cuadernos $U C A B$ N. 3, 113-118. Recuperado de http://biblioteca2.ucab.edu.ve/ anexos/biblioteca/marc/texto/AAM0182_3.pdf

Bourdieu, P. \& Passeron, J.-C., (2009). Los herederos: Los estudiantes y la cultura. Buenos Aires: Siglo XXI.

Dávila Quintero, J. A. Núñez, L. A., Sandia Saldivia, B. E., Torréns Heeren, R. (2006). Los repositorios institucionales y la preservación del patrimonio intelectual académico, Interciencia, 31, 1, 22-28.

Devés Valdés, E. (2006), Las tareas de intelectuales y universitarios ante el estancamiento de América Latina hacia las redes y la internacional del conocimiento. Recuperado de http:/ / umbral. uprrp.edu/?q=Las_tareas_de_intelectuales_y_universitarios _ante_el_estancamiento_de_America_Latina

Downes, S. (2011). MOOC 2011: The Massive Open Online Course in Theory and in Practice. Recuperado de http://www.slideshare. net/Downes/xmooc-the-massive-open-online-course-intheory-and-in-practice

Granell de Aldaz, E., Garaway, D. \& Malpica, C. (1997). Éxito gerencial y cultura: retos y oportunidades en Venezuela. Caracas: Ediciones IESA.

Lareau, A. (2003). Unequal childhoods: class, race, and family life. California: University of California Press.

Leistner, F. (2010). Mastering organizational knowledge flow: how to make knowledge sharing work. New Jersey: Wiley and SAS. 
Martínez García, J. S. (1998). Las clases sociales y el capital en Pierre Bourdieu: Un intento de aclaración (comunicación presentada en el VI Congreso de Sociología de la FES). Recuperado de http://josamaga.webs.ull.es/Papers/clasebd-usal.pdf

Morales, J. T. (2014). La construcción teórica en las tesis doctorales de Ciencias Sociales Educación, Revista ARJÉ, 8(14), 233-249.

Oppenheimer, A. (2012). Cuáles son los países más conectados. Recuperado de http://www.lostiempos.com/diario/opiniones /columnistas/20120410/los-paises-mas-conectados_167250 _350889.html

Ordóñez V., L. A. (2011). La inserción transversal de la conectividad en el currículum de los estudios generales. Ciencia y Sociedad, 36(3), 450-463. Recuperado de http://issuu.com/lordonezv/ docs/named82ad4

Ordóñez, V., L. A. (2012). Cultura participativa y conectivismo: algunos retos para la investigación latinoamericana, en Diálogos y desafíos euro-latinoamericanos: Ensayos sobre cooperación, derecho, educación y comunicación. (Elías Said Hung, Ed). Universidad del Norte, Barranquilla, Colombia.

Rial, A. (1997). La variable independiente: el rol de la idiosincrasia y los valores sociales en el desarrollo de Venezuela. Caracas: Galac.

Roche, M. (1975). La politica de la ciencia en Venezuela (serie Descubriendo a Prometeo). Caracas: Monte Ávila Editores.

Siemens, G. (2004). Connectivism: A Learning Theory for the Digital Age. Recuperado http://www.elearnspace.org/Articles/ connectivism.htm 
Volek, E. (2007). Anverso y reverso del laberinto de la soledad: Octavio Paz y cien años de Macondo. Cuadernos del CILHA, 8(9), 131-143. Recuperado de http://www.redalyc.org/ pdf/1817/181715655012.pdf

Williams, R., T., Mackness, J. \& Gumtau, S. (2012). Footprints of emergence: The International Review of Research in Open and Distance Learning (IRRODL), 13(4), 49-90.

Willis, P. (1977). Learning to labor: How working class kids get working class jobs. New York: Columbia University Press.

\section{Dr. Luis Arnaldo Ordóñez Vela}

Nacido en Maracay, Venezuela. Tiene una licenciatura en Química por la Universidad Central de Venezuela; doctor en Bioquímica egresado del Instituto Tecnológico de Massachusetts, Estados Unidos; culminó el Programa Avanzado de Gerencia (PAG) en el Instituto de Estudios Superiores de Administración (IESA), de Caracas, Venezuela.

Sus intereses actuales de investigación giran alrededor de los determinantes de la participación en la sociedad y el impacto que tienen en la misma la presencia acelerada de las tecnologías digitales, así como el rol que juega la información en los mecanismos decisionales en nuestro medio latinoamericano y la movilización de conocimientos entre la universidad y la sociedad latinoamericana. 
La movilización de conocimientos en la sociedad:

Un reto para los programas doctorales en la universidad pública latinoamericana

Sus publicaciones recientes son:

- Aprendiendo a aprender en la web: Una introducción al aprendizaje conectivista.

- Información o comunicación: el falso dilema de las bibliotecas universitarias.

- La promesa de internety la participación ciudadana.

- Cultura participativa y conectivismo en la educación superior.

- Los partidos políticos venezolanos y su estrategia de aproximación a internet: una mirada no tecnológica.

- Variables psicosociales y microcréditos como estrategias de luchas contra la pobreza, entre muchas otras.

Correo electrónico: lordonezv@gmail.com

Recibido: 15-7-2015

Aprobado: 24-8-2015

Ciencia y Sociedad 2015; 40(4): 685-723 723 
